\title{
A computational approach to Lüroth quartics
}

\author{
Giorgio Ottaviani*
}

\begin{abstract}
A plane quartic curve is called Lüroth if it contains the ten vertices of a complete pentalateral. White and Miller constructed in 1909 a covariant quartic 4-fold, associated to any plane quartic. We review their construction and we show how it gives a computational tool to detect if a plane quartic is Lüroth. As a byproduct, the 28 bitangents of a general plane quartic correspond to 28 singular points of the associated White-Miller quartic 4-fold.
\end{abstract}

\section{Introduction}

A Lüroth quartic is a plane quartic containing the ten vertices of a complete pentalateral, like in Figure 1. The pentalateral is inscribed in the quartic, and (equivalently) the quartic circumscribes the pentalateral. The Lüroth

${ }^{*}$ The author is member of GNSAGA-INDAM.

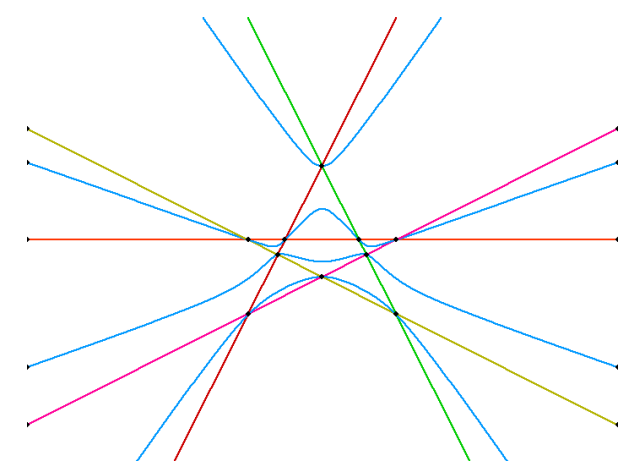

Figure 1: A Lüroth quartic with a pentalateral inscribed, plotted with XAlci. 
quartics attracted a lot of attention in the classical literature, because they show a "poristic" phenomenon, namely "the fallacy of constant counting", to use the words of White and Miller, [22].

Indeed, Lüroth was the first to observe, in 1868, that when a plane quartic circumscribes one pentalateral, it also circumscribes infinitely many other pentalaterals, which is not expected by a naive dimension count (see [22] pag. 348). As a consequence of Lüroth's result, Lüroth quartics are not dense in the space of quartics, but they fill an open subset of a hypersurface of $\mathbb{P}^{14}$. The equation of this hypersurface is called the Lüroth invariant and, to the best of our knowledge, it is still unknown. This hypersurface consists entirely of quartics, so the limits (when the pentalateral degenerates) are also called nowadays Lüroth quartics. Lüroth found a one dimensional family of inscribed pentalaterals, all tangent to the same conic. There are examples with more families of pentalaterals (see §4), and so more conics. Each family defines a particular even theta characteristic, called pentalateral theta. Let $\delta$ be the number of pentalateral theta on a general Lüroth quartic. It is a well-established fact, already known to classical geometers, that the Lüroth invariant has degree $54 / \delta$ (see the proof of Theorem 5.1). The classical study on Lüroth quartics culminated in 1919 in Morley's proof[11] that the Lüroth invariant has degree 54 . We reviewed this lovely proof in a joint paper with $\mathrm{E}$. Sernesi [12], and we refer to [12, 4, 9, 21] for more information, including the role of Lüroth quartics in vector bundle theory. In equivalent way, Morley proved that $\delta=1$, that is that for the general Lüroth quartic there is a unique family of inscribed pentalaterals, all tangent to the same conic, which is uniquely determined. We emphasized this point of view, even if it was not focal in [11, because it is the starting point of our current paper.

We try to answer to the following

Question: given an explicit quartic curve given by a homogeneous quartic polynomial in three variables, how can one detect if it is Lüroth?

The answer should follow from the explicit expression of the Lüroth invariant. Since its expression is unclear, we have to look for alternative roads.

For this purpose, we review a construction due to White and Miller [22]. They construct, for any plane quartic curve $f$, a covariant quartic 4 -fold $\mathrm{WM}_{f}$ in the space $\mathbb{P}^{5}$ of conics. They proved that when $f$ is Lüroth with a pentalateral theta corresponding to the conic $q$, the point $q \in \mathbb{P}^{5}$ is a singular point of $\mathrm{WM}_{f}$. White-Miller's aim was to use this property to characterize the Lüroth property, with the hope that the discriminant of $\mathrm{WM}_{f}$ could be a power of the Lüroth invariant. Unfortunately, this attempt fails, because 
the discriminant of $\mathrm{WM}_{f}$ vanishes identically.

Indeed we prove

Theorem 0.1 For the general plane quartic $f, \mathrm{WM}_{f}$ has always 28 singular points corresponding to the 28 bitangents (odd theta characteristics) of $f$.

This theorem owes a lot to the computer experiments performed with Macaulay2 [7]. The failure of White-Miller's hope is balanced by two remarks, which give,in some sense, a reprise to that hope, and show that the quartic $\mathrm{WM}_{f}$ is an interesting object. The first one is that Theorem 0.1 gives a way to compute the ideal of the bitangents, and it gives at once the information about the bitangent lines and the tangency points.

The second remark is that it allows one to partially answer our question of how to detect if a quartic is Lüroth. Indeed, with Macaulay2, it is possible to quickly compute the singular locus of $\mathrm{WM}_{f}$, given any $f \in S^{4} V$. The following outcomes are possible

-(i) if the singular locus of $\mathrm{WM}_{f}$ has dimension zero and degree 28 , then $f$ is not Lüroth. This outcome is the general one.

-(ii) if the singular locus of $\mathrm{WM}_{f}$ has dimension zero and degree 29, the "extra" point can be found by quotient over the ideal generated by the cubic hypersurface of singular conics. When it remains a single point, it corresponds to a smooth conic. In this case, after the check of a mild open condition (see \$4), $f$ is Lüroth and the conic corresponds to the unique pentalateral theta. This outcome is found for the general Lüroth curve $f$. In this case the explicit equation of all the pentalaterals inscribed can be found.

-(iii) if the outcome is different from (i) and (ii) a further analysis is necessary. The double irreducible conics, which are not Lüroth (see the proof of Prop. 4.1 in [13]), lie in this third category. If $f$ is a double irreducible conic, then the singular locus of $\mathrm{WM}_{f}$ consists of a surface of degree 10, whose general point is a singular conic, plus a curve of degree 4, whose general point corresponds to a smooth conic. Also the desmic quartics, which are Lüroth and have at least 6 families of inscribed pentalateral (see [2] pag. 367), lie in this third category. When $f$ is desmic, $\mathrm{WM}_{f}$ is non reduced and it is a double hyperquadric in $\mathbb{P}^{5}$ (see Section 6).

As a consequence we get a computer-aided proof that the Lüroth invariant has degree 54. This proof is conceptually simpler than the two known proofs, respectively by Morley[11, 12] and by LePotier-Tikhomirov[9], but it cannot be concluded without the help of a computer. 
The material collected here owes a lot to the many thorough discussions I had on the topic with Edoardo Sernesi during the preparation of [12] and [13. It is a pleasure to thank Edoardo for his insight and method. I thank also Igor Dolgachev and Bernd Sturmfels for their interest and many useful comments.

\section{Apolarity and the cubic invariant of plane quartics}

Given any complex vector space $U$, we denote by $U^{\vee}$ its dual space. Let $S^{d} U$ be the $d$-th symmetric power of $U$, its elements are homogeneous polynomials of degree $d$ in any coordinate system for $U$. Any $f \in S^{d} U$ may be identified (up to scalar multiples) with its zero scheme in the projective space $\mathbb{P}(U)$ of hyperplanes in $U$, so $f$ denotes also a hypersurface of degree $d$.

We recall a few facts about apolarity [6, 15]. A polynomial $f \in S^{d} U$ is called apolar to a polynomial $g \in S^{e} U^{\vee}$ if the contraction $f \cdot g \in S^{d-e} U$ is zero. It is convenient to consider $g$ as a differential operator acting over $f$. In the case $d=e$, the symmetric convention, that $f$ acts over $g$, works as well.

When $\operatorname{dim} U=2$, that is for polynomials over a projective line, the apolarity is well defined for $f, g$ both in $S^{d} U$. This is due to the canonical isomorphism $U \simeq U^{\vee} \otimes \wedge^{2} U$. Let $\left(x_{0}, x_{1}\right)$ be coordinates on $U$. If $f=\left(a_{0} x_{0}+a_{1} x_{1}\right)^{d}$ and $g=\left(b_{0} x_{0}+b_{1} x_{1}\right)^{d}$, then the contraction between $f$ and $g$ is easily seen to be proportional to $\left(a_{0} b_{1}-a_{1} b_{0}\right)^{d}$. This computation extends by linearity to any pair $f, g \in S^{d} U$, because any polynomial can be expressed as a sum of $d$-th powers. The resulting formula for $f=\sum_{i=0}^{d}\left(\begin{array}{l}d \\ i\end{array}\right) f_{i} x^{d-i} y^{i}$ and $g=\sum_{i=0}^{d}\left(\begin{array}{c}d \\ i\end{array}\right) g_{i} x^{d-i} y^{i}$ is that $f$ is apolar to $g$ if and only if

$$
\sum_{i=0}^{d}(-1)^{i}\left(\begin{array}{l}
d \\
i
\end{array}\right) f_{i} g_{d-i}=0
$$

In particular

Lemma 1.1 Let $p, l^{d} \in S^{d} U . p$ is apolar to $l^{d}$ if and only if $l$ divides $p$.

A polynomial $f \in S^{4} U$ is called equianharmonic if its apolar to itself. So $f$ is equianharmonic if and only if

$$
f_{0} f_{4}-4 f_{1} f_{3}+3 f_{2}^{2}=0
$$


which is the expression for the classical invariant $I$ of binary quartics.

Let $\left(x_{0}, x_{1}, x_{2}\right)$ be coordinates on a 3 -dimensional complex space $V$ and $\left(y_{0}, y_{1}, y_{2}\right)$ be coordinates on $V^{\vee}$. Let

$$
f\left(x_{0}, x_{1}, x_{2}\right)=\sum_{i+j+k=4} \frac{4 !}{i ! j ! k !} f_{i j k} x_{0}^{i} x_{1}^{j} x_{2}^{k} \in S^{4} V
$$

be the equation of a plane quartic curve on $\mathbb{P}(V)$. All invariants of $f$ have degree which is multiple of $3([5,20])$. The invariant of smallest degree has degree 3 and it corresponds to a trilinear form $A(f, g, h)$, for $f, g, h \in S^{4} V$. It is defined as

$$
A\left(\left(a_{0} x_{0}+a_{1} x_{1}+a_{2} x_{2}\right)^{4},\left(b_{0} x_{0}+b_{1} x_{1}+b_{2} x_{2}\right)^{4},\left(c_{0} x_{0}+c_{1} x_{1}+c_{2} x_{2}\right)^{4}\right)=\left|\begin{array}{ccc}
a_{0} & a_{1} & a_{2} \\
b_{0} & b_{1} & b_{2} \\
c_{0} & c_{1} & c_{2}
\end{array}\right|^{4}
$$

This definition extends by linearity to any $f, g, h \in S^{4} V$. The explicit expression of the cubic invariant $A$ can be found at art. 293 of Salmon's book [16, it can be checked today e.g. with Macaulay2 ([7]) and it is the sum of the following 23 terms (we denote $A(f)$ for $A(f, f, f)$ )

$$
\begin{gathered}
A(f)=f_{400} f_{040} f_{004}+3\left(f_{220}^{2} f_{004}+f_{202}^{2} f_{040}+f_{400} f_{022}^{2}\right)+12\left(f_{202} f_{121}^{2}+f_{220} f_{112}^{2}+f_{022} f_{211}^{2}\right)+6 f_{220} f_{202} f_{022}+ \\
-4\left(f_{301} f_{103} f_{040}+f_{400} f_{031} f_{013}+f_{310} f_{130} f_{004}\right)+4\left(f_{310} f_{103} f_{031}+f_{301} f_{130} f_{013}\right)+ \\
-12\left(f_{202} f_{130} f_{112}+f_{220} f_{121} f_{103}+f_{211} f_{202} f_{031}+f_{301} f_{121} f_{022}+f_{310} f_{112} f_{022}+f_{220} f_{211} f_{013}+f_{211} f_{121} f_{112}\right)+ \\
+12\left(f_{310} f_{121} f_{013}+f_{211} f_{130} f_{103}+f_{301} f_{112} f_{031}\right)
\end{gathered}
$$

We will see in Remark 1.3 a more geometric way to recover the same expression.

We will need in the sequel another expression for the cubic invariant, borrowed from [22]. Call $\bar{f}=\left(f_{400}, \ldots, f_{004}\right)^{t}$ the (column) vector of coefficients of $f$. The trilinear form can be written in the form

$$
A(f, g, h)=\bar{f}^{t} L_{g} \bar{h}
$$

where $L_{g}$ is a $15 \times 15$ symmetric matrix with entries linear in $g$. The matrix $L_{g}$ encodes all the information to express the cubic invariant. It is denoted $L$ at page 349 of [22], its explicit expression is reported in the appendix .

Note that given $f, g \in S^{4} V$, the equation $A(f, g, *)=0$ defines an element in the dual space $S^{4} V^{\vee}$, possibly vanishing. 
Proposition 1.2 (i) $A\left(f, g, l^{4}\right)=0$ if and only if the restrictions $f_{\mid l}, g_{\mid l}$ to the line $l=0$ are apolar.

(ii) Let $A(f, g, *)=H$. We have $A\left(f, g, l^{4}\right)=0$ if and only if $H(l)=0$.

Proof. To prove (i), consider $f=\left(\sum_{i=0}^{2} a_{i} x_{i}\right)^{4}, g=\left(\sum_{i=0}^{2} b_{i} x_{i}\right)^{4}, l=x_{2}$. Then

$$
A\left(f, g, l^{4}\right)=\left|\begin{array}{ccc}
a_{0} & a_{1} & a_{2} \\
b_{0} & b_{1} & b_{2} \\
0 & 0 & 1
\end{array}\right|^{4}=\left|\begin{array}{cc}
a_{0} & a_{1} \\
b_{0} & b_{1}
\end{array}\right|^{4}=f_{\mid l} \cdot g_{\mid l}
$$

This formula extends by linearity to any $f, g$.

(ii) follows because $H(l)=H \cdot l^{4}$.

Remark 1.3 The part (i) of Proposition 1.2 gives an alternative way to define $L_{g}$. Let $l$ with equation $\sum_{i=0}^{2} x_{i} y_{i}=0$. Substitute in $f$ and $g$ the expression $-\frac{y_{1}}{y_{0}} x_{1}-\frac{y_{2}}{y_{0}} x_{2}$ at the place of $x_{0}$, after getting rid of the denominators we may define $\tilde{f}\left(x_{1}, x_{2}\right)=f\left(-y_{1} x_{1}-y_{2} x_{2}, y_{0} x_{1}, y_{0} x_{2}\right)$ and $\tilde{g}\left(x_{1}, x_{2}\right)=$ $g\left(-y_{1} x_{1}-y_{2} x_{2}, y_{0} x_{1}, y_{0} x_{2}\right)$.

Then, the expression

$$
\tilde{f}_{04} \tilde{g}_{40}-4 \tilde{f}_{13} \tilde{g}_{31}+6 \tilde{f}_{22} \tilde{g}_{22}-4 \tilde{f}_{31} \tilde{g}_{13}+\tilde{f}_{40} \tilde{g}_{04}
$$

is equivalent to $y_{0}^{4} A\left(f, g, l^{4}\right)$. Differentiating by the coefficients of $l^{4}$ and $f$ one finds easily $L_{g}$. It is interesting that the whole expression (3) is divisible by $y_{0}^{4}$, while its individual summands are not.

Remark 1.4 Let $l_{1}, l_{2}$ be two lines. $A\left(l_{1}^{4}, l_{2}^{4}, f\right)=0$ gives the condition that $f$ passes through the intersection point $l_{1}=l_{2}=0$.

Note also from Prop. 1.2 that $A\left(f, f, l^{4}\right)=0$ if and only if $f$ cuts $l$ in an equianharmonic 4-tuple. The quartic curve $A(f, f, *)$ in the dual space is called the equianharmonic envelope of $f$, and consists of all lines which are cut by $f$ in a equianharmonic 4-tuple .

This gives the classical geometric interpretation of the cubic invariant for plane quartics. The condition $A(f, f, f)=0$ means that $f$ is apolar with its own equianharmonic envelope (see [3]), note that it gives a solution to Exercise (1) in the last page of [20]. 


\section{Clebsch and Lüroth quartics}

A plane quartic $f \in S^{4} V$ is called Clebsch if it has an apolar conic, that is if there exists a nonzero $q \in S^{2} V^{\vee}$ such that $q \cdot f=0$.

One defines, for any $f \in S^{4} V$, the catalecticant map $C_{f}: S^{2} V^{\vee} \rightarrow S^{2} V$ which is the contraction by $f$.

The equation of the Clebsch invariant is easily to be seen as the determinant of $C_{f}$, that is we have([6], example (2.7))

Theorem 2.1 (Clebsch) A plane quartic $f$ is Clebsch if and only if $\operatorname{det} C_{f}=$ 0 . The conics which are apolar to $f$ are the elements of $\operatorname{ker} C_{f}$.

It follows ([4], Lemma 6.3.22) that the general Clebsch quartic can be expressed as a sum of five 4 -th powers, that is

$$
f=\sum_{i=0}^{4} l_{i}^{4}
$$

A general Clebsch quartic $f$ can be expressed as a sum of five 4-th powers in $\infty^{1}$ many ways. Precisely the 5 lines $l_{i}$ belong to a unique smooth conic $Q$ in the dual plane, which is apolar to $f$ and it is found as the generator of ker $C_{f}$. Equivalently, the 5 lines $l_{i}$ are tangent to a unique conic, which is the dual conic of $Q$.

We recall that a theta characteristic on a general plane quartic $f$ is a line bundle $\theta$ on $f$ such that $\theta^{2}$ is the canonical bundle. Hence $\operatorname{deg} \theta=2$. There are 64 theta characteristic on $f$. If the curve is general, every bitangent is tangent in two distinct points $P_{1}$ and $P_{2}$, and the divisor $P_{1}+P_{2}$ defines a theta characteristic $\theta$ such that $h^{0}(\theta)=1$, these are called odd theta characteristic and there are 28 of them. The remaining 36 theta characteristic $\theta$ are called even and they satisfy $h^{0}(\theta)=0$.

The Scorza map is the rational map from $\mathbb{P}^{14}=\mathbb{P}\left(S^{4} V\right)$ to itself which associates to a quartic $f$ the quartic $S(f)=\left\{x \in \mathbb{P}(V) \mid A r\left(P_{x}(f)\right)=0\right\}$, where $P_{x}(f)$ is the cubic polar to $f$ at $x$ and $A r$ is the Aronhold invariant [19, 6, 4]. A convenient way to write explicitly the Scorza map is through the expression of the Aronhold invariant in [8], example 1.2.1. It is well known that the Scorza map is a $36: 1$ map. Indeed the curve $S(f)$ is equipped with an even theta characteristic. For a general quartic curve, its 36 inverse images through the Scorza map give all the 36 even theta characteristic on $S(f)$. 
A Lüroth quartic is a plane quartic containing the ten vertices of a complete pentalateral, or the limit of such curves.

If $l_{i}$ for $i=0, \ldots, 4$ are the lines of the pentalateral, we may consider them as divisors (of degree 4 ) over the curve. Then $l_{0}+\ldots+l_{4}$ consists of 10 double points, the meeting points of the 5 lines. Let $P_{1}+\ldots+P_{10}$ the corresponding reduced divisor of degree 10. Then $P_{1}+\ldots+P_{10}=2 H+\theta$ where $H$ is the hyperplane divisor and $\theta$ is a even theta characteristic, which is called the pentalateral theta. The pentalateral theta was called pentagonal theta in [6], and it coincides with [4], Definition 6.3.30 (see the comments thereafter).

The following result is classical[19], for a modern proof see [6, 4].

Proposition 2.2 Let $f$ be a Clebsch quartic with apolar conic $Q$, then $S(f)$ is a Lüroth quartic equipped with the pentalateral theta corresponding to $Q$.

The number of pentalateral theta on a general Lüroth quartic, called $\delta$ in the introduction, is equal to the degree of the Scorza map when restricted to the hypersurface of Clebsch quartics.

Explicitly, if $f$ is Clebsch with equation

$$
l_{0}^{4}+\ldots+l_{4}^{4}=0
$$

then $S(f)$ has equation

$$
\sum_{i=0}^{4} k_{i} \prod_{j \neq i} l_{j}=0
$$

where $k_{i}=\prod_{p<q<r, i \notin\{p, q, r\}}\left|l_{p} l_{q} l_{r}\right|$ (see [4], Lemma 6.3.26) so that $l_{0}, \ldots, l_{4}$ is a pentalateral inscribed in $S(f)$. Note that the conic where the five lines which are the summands of $f$ are tangent, is the same conic where the pentalateral inscribed in $S(f)$ is tangent.

The starting point of White-Miller paper [22] is the following remarkable characterization.

Proposition 2.3 Let $f$ be a general Clebsch quartic and let $Q$ be the conic apolar to $f$. Let $L$ be a line. $L$ belongs to $Q \Longleftrightarrow$ the two binary quartic forms $f_{\mid L}$ and $S(f)_{\mid L}$ are apolar.

Proof. $\Longrightarrow$ Let $L=l_{4}$ in the expression (4). Then $f_{\mid L}=\sum_{i=0}^{3} l_{i}^{4}$ and $S(f)_{\mid L}=$ $\prod_{i=0}^{3} l_{i}$. So the result is immediate by the Lemma 1.1 . 
$\Longleftarrow$ The proof is an explicit computation. Let $f=\sum_{i=0}^{4}\left(\alpha_{0 i} x_{0}+\alpha_{1 i} x_{1}+\right.$ $\left.\alpha_{2 i} x_{2}\right)^{4}$. Let $x_{0}=0$ be the equation of $L$. The condition that $L$ belongs to $Q$ can be expressed as the vanishing of the degree 10 polynomial in the $\alpha_{i j}$ given by the determinant

$$
D\left(\ldots, \alpha_{i j}, \ldots\right)=\left|\begin{array}{ccccc}
1 & 0 & 0 & 0 & 0 \\
\alpha_{00}^{2} & \alpha_{00} \alpha_{10} & & & \alpha_{20}^{2} \\
\alpha_{01}^{2} & \alpha_{01} \alpha_{11} & & & \alpha_{21}^{2} \\
\vdots & \vdots & & & \vdots \\
\alpha_{04}^{2} & \alpha_{04} \alpha_{14} & & & \alpha_{24}^{2}
\end{array}\right|
$$

Let $g=S(f), \tilde{f}=f\left(0, x_{1}, x_{2}\right), \tilde{g}=g\left(0, x_{1}, x_{2}\right)$. The condition that $f_{\mid L}$ and $S(f)_{\mid L}$ are apolar can be expressed as the vanishing of the degree 20 polynomial

$$
P\left(\ldots, \alpha_{i j}, \ldots\right)=\tilde{f}_{04} \tilde{g}_{40}-4 \tilde{f}_{13} \tilde{g}_{31}+6 \tilde{f}_{22} \tilde{g}_{22}-4 \tilde{f}_{31} \tilde{g}_{13}+\tilde{f}_{40} \tilde{g}_{04}
$$

By the previous implication, we already know that $D$ divides $P$. An explicit computation with Macaulay2 shows that (up to scalar multiples) $D\left(\ldots, \alpha_{i j}, \ldots\right)^{2}=$ $P\left(\ldots, \alpha_{i j}, \ldots\right)$. This proves both the implications at once.

\section{The White-Miller quartic $\mathrm{WM}_{f}$}

Proposition 3.1 Assume $f, g \in S^{4} V$ and $\operatorname{det} L_{g} \neq 0$ (see (2). Let's define $H \in S^{4} V^{\vee}$ by $H=A(f, g, *)$. Then $f$ can be recovered by $g$ and $H$ as the expression of the following bordered determinant

$$
f(x)=\left|\begin{array}{cc}
L_{g} & \bar{H} \\
(x)^{4} & 0
\end{array}\right|
$$

where $(x)^{4}$ denotes the 4 -th symmetric power of the row vector $\left(x_{0}, x_{1}, x_{2}\right)$ containing all terms of the form $\frac{4 !}{i ! j ! k !} x_{0}^{i} x_{1}^{j} x_{2}^{k}$ for $i+j+k=4$.

Proof. The hypothesis reads as $L_{g} \bar{f}=\bar{H}$. The assertion reads as $\bar{f}=L_{g}^{-1} \bar{H}$.

Proposition 3.2 (White-Miller) Let $f \in S^{4} V$ be a Clebsch quartic with apolar conic $Q \in S^{2} V^{\vee}$. Let $g=S(f)$ be the associated Lüroth quartic (see Prop. 2.2). Then, up to scalar multiples, $A(f, S(f), *)=Q^{2} \in S^{4} V^{\vee}$. 
Proof. It is a reformulation of Prop. 2.3 and Prop. 1.2 .

Corollary 3.3 Let $f$ be a Clebsch quartic with apolar conic $Q$ and $g=S(f)$ be Lüroth.

(i) If det $L_{g} \neq 0$, then the expression of $f$ can be recovered, up to scalar multiples, from $g$ and $Q$ by the formula

$$
f=\left|\begin{array}{cc}
L_{g} & \overline{Q^{2}} \\
(x)^{4} & 0
\end{array}\right|
$$

(ii) If $\operatorname{det} L_{f} \neq 0$, then the expression of $g$ can be recovered, up to scalar multiples, from $f$ and $Q$ by the formula

$$
g=\left|\begin{array}{cc}
L_{f} & \overline{Q^{2}} \\
(x)^{4} & 0
\end{array}\right|
$$

Corollary 3.4 Let $g$ be a Lüroth quartic with pentalateral theta corresponding to the conic $Q$. Then the following identity holds for every $(x)^{2}=$ $\left(x_{0}^{2}, \ldots, x_{2}^{2}\right)$

$$
\left|\begin{array}{cc}
L_{g} & \overline{Q^{2}} \\
(x)^{2} \bar{Q}^{t} & 0
\end{array}\right|=0
$$

Proof. Write that $f \cdot Q=0$ in Corollary 3.3 .

The previous corollary gives the motivation for the following definition.

Definition 3.5 For any $f \in S^{4} V$, the White-Miller quartic $\mathrm{WM}_{f}$ is defined in $\mathbb{P}\left(S^{2} V\right)$ with coordinates $\left(q_{0}, \ldots q_{5}\right)$ by the formula

$$
\mathrm{WM}_{f}=\left|\begin{array}{cc}
L_{f} & \left(q^{2}\right)^{t} \\
q^{2} & 0
\end{array}\right|
$$

where $\left(q^{2}\right)$ is the vector of the 15 cooefficients of a double conic. Note that $\mathrm{WM}_{f}$ corresponds to the unique irreducible summand isomorphic to $S^{4} V^{\vee}$ inside $S^{4}\left(S^{2} V\right)$.

The definition is taken verbatim from [22, it is equivalent to

$$
\mathrm{WM}_{f}=q^{2}\left(\operatorname{ad} L_{f}\right)\left(q^{2}\right)^{t}
$$

where $\operatorname{ad} L_{f}$ is the adjoint matrix of $L_{f}$. 
Proposition 3.6 (White-Miller) Let $f$ be a Lüroth quartic with pentalateral theta corresponding to the conic $Q$. Then $Q$ is a singular point of $\mathrm{WM}_{f}$.

Proof. The six partial derivatives of

$$
\left|\begin{array}{cc}
L_{f} & q^{2} \\
q^{2} & 0
\end{array}\right|
$$

computed in the point $Q$ vanish if and only if

$$
\left|\begin{array}{cc}
L_{g} & \overline{Q^{2}} \\
(x)^{2} \bar{Q}^{t} & 0
\end{array}\right|=0
$$

for every $(x)^{2}$. This identity holds by Corollary 3.4 .

If $\mathrm{WM}_{f}$ is not singular elsewhere, then the discriminant of $\mathrm{WM}_{f}$ should detect if $f$ is Lüroth. This was the aim of White-Miller construction. In the next section we see that there are at least other 28 singular points for $\mathrm{WM}_{f}$, so that its discriminant vanish identically.

\section{The proof of Theorem 0.1 and the algo- rithm to detect if a quartic is Lüroth}

Proposition 4.1 Let $f, l^{4} \in S^{4} V$ and consider $A\left(f, l^{4}, *\right) \in S^{4} V^{\vee}$ as a quartic in $\mathbb{P}\left(V^{\vee}\right)$.

(i) $A\left(f, l^{4}, *\right)$ splits in four lines concurrent lines, corresponding to the four intersection points where $f$ and $l$ meet.

(ii) $A\left(f, l^{4}, *\right)$ is a double conic if and only if $l$ is a bitangent to $f$.

Proof. (i) follows from Prop. 1.2 (ii) and Remark 1.4. (ii) is immediate from (i).

Definition 4.2 Let $l$ be a bitangent to $f$. It defines a reducible conic $Q_{l, f}$ in $\mathbb{P}\left(V^{\vee}\right)$ given by the two pencils through the two points of tangency. On the algebraic side we have, from Prop. 4.1. (ii), the equivalent definition from the identity (up to scalar multiples)

$$
Q_{l, f}^{2}=A\left(f, l^{4}, *\right)
$$


The following Proposition proves the Theorem 0.1 .

Proposition 4.3 Let $f \in S^{4} V$ and $\operatorname{det} L_{f} \neq 0$. Let $l$ be a bitangent to $f$ and let $Q_{l, f}$ be the corresponding reducible conic of Def. 4.2.

The quartic $\mathrm{WM}_{f}$ is singular in $Q_{l, f}$.

Proof. From Prop. 3.1 it follows that $l^{4}$ is the expression of the bordered determinant

$$
\left|\begin{array}{cc}
L_{f} & \overline{Q_{l, f}^{2}} \\
(x)^{4} & 0
\end{array}\right|
$$

Since $Q_{l, f}$ splits in two pencils of lines through points of $l$, we get the relation $Q_{l, f} \cdot l^{4}=0$, it follows

$$
\left|\begin{array}{cc}
L_{f} & \overline{Q_{l, f}^{2}} \\
(x)^{2} \overline{Q_{l, f}} & 0
\end{array}\right|=0 \quad \forall(x)^{2}
$$

that implies the thesis.

The algorithm sketched in the introduction, based on the analysis of the singular locus of $\mathrm{WM}_{f}$, works as follows.

-(i) if the singular locus of $\mathrm{WM}_{f}$ has dimension zero and degree 28, then $f$ is not Lüroth because otherwise the pentalateral theta should give a 29th singular point by Prop. 3.6.

-(ii) if the singular locus of $\mathrm{WM}_{f}$ contains a point corresponding to a smooth conic $Q$, then by Prop. 3.1 the formula

$$
g=\left|\begin{array}{cc}
L_{f} & Q^{2} \\
(x)^{4} & 0
\end{array}\right|
$$

defines a quartic which is apolar to $Q$, hence it is Clebsch.

If det $L_{g} \neq 0$, from Corollary 3.3 it follows that

$$
f=\left|\begin{array}{cc}
L_{g} & Q^{2} \\
(x)^{4} & 0
\end{array}\right|
$$

and in particular $f$ coincides with $S(g)$ and it is Lüroth.

Moreover, assume that the rank of the catalecticant $C_{g}$ is 5 . Fixing a line $l_{0}$ which belongs to $Q$, we get that $g^{\prime}=g-\alpha_{0} l_{0}^{4}$ has rank 4 for a convenient $\alpha_{0} \in \mathcal{C}$. The kernel of the catalecticant $C_{g^{\prime}}$ is generated by two conics, which 
meet in 4 points, which correspond to $l_{1}, \ldots, l_{4}$. By a classical technique, which goes back to Sylvester, there exist scalars $\alpha_{i}$ for $i=1, \ldots, 4$ such that $g=\sum_{i=0}^{4} \alpha_{i} l_{i}^{4}([15])$ and $l_{i}$ for $i=0, \ldots, 4$ give a pentalateral inscribed to $f$.

-(iii) In the case where the singular locus of $\mathrm{WM}_{f}$ is bigger than 29 points, or in the case (ii) when $\operatorname{det} L_{g}=0$, a further analysis is necessary and only partial results can be achieved by our algorithm. Some examples in this third category, either Lüroth or not Lüroth, have been analyzed at the end of the introduction. Note that if $f$ is a desmic quartic then $\operatorname{det} L_{f}=0$ and Corollary 3.3 does not apply. More examples are in the Section 5 .

\section{Computational facts and examples}

Theorem 5.1 (Morley) The Lüroth invariant has degree 54.

Proof.

The Scorza map is a rational map from $\mathbb{P}^{14}$ to $\mathbb{P}^{14}$ with entries of degree 4 in the 15 homogeneous coefficients of a quartic $f$. We call $L$ the degree of the Lüroth invariant. From the discussion after Prop. 2.2, since the Clebsch invariant has degree 6 we get the well known equality

$$
\frac{4 L}{36 / \delta}=6
$$

hence $54=\delta L$. This equality coincides with the equation (2.5) in [21] specialized to $r=5, d=4$, where $s_{4}(5)=54$ (see [21] $\left.\S 5\right), \operatorname{deg} p_{C}$ is our $\delta$ and $p_{C}\left(\mathrm{MP}_{5}^{4}\right)$ is the Lüroth hypersurface in $\mathbb{P}^{14}$.

If

$f=x_{0} x_{1} x_{2}\left(x_{0}+x_{1}+x_{2}\right)+\left(x_{0}+2 x_{1}+3 x_{2}\right)\left(x_{0} x_{1} x_{2}+\left(x_{0} x_{1}+x_{0} x_{2}+x_{1} x_{2}\right)\left(x_{0}+x_{1}+x_{2}\right)\right)$

then a Macaulay2 computation says thatWM $\mathrm{M}_{f}$ has exactly 29 singular points. This implies that the Lüroth quartic $f$ has a unique pentalateral theta, corresponding to the conic tangent to the pentalateral given by $l_{0}=x_{0}, l_{1}=x_{1}$, $l_{2}=x_{2}, l_{3}=x_{0}+x_{1}+x_{2}, l_{4}=x_{0}+2 x_{1}+3 x_{2}$. By semicontinuity, the general Lüroth quartic curve has a unique pentalateral theta. This implies $\delta=1$ and the theorem follows.

In the example $f=x_{2}^{2}\left(x_{0}^{2}+x_{1}^{2}\right)+x_{2}\left(x_{0}^{3}+x_{1}^{3}\right)-x_{0}^{3} x_{1}+(1 / 2) x_{0}^{2} x_{1}^{2}-x_{0} x_{1}^{3}$, found in [9] section 9.3 (put $c=-\frac{1}{4}$ ), the singular locus of $\mathrm{WM}_{f}$ consists of 
a conic, whose general point corresponds to a singular conic, plus 8 points, each one corresponding to a smooth conic. This example has 8 distinct pentalateral theta.

In the example $f=25\left(x_{0}^{4}+x_{1}^{4}+x_{2}^{4}\right)-34\left(x_{0}^{2} x_{1}^{2}+x_{0}^{2} x_{2}^{2}+x_{1}^{2} x_{2}^{2}\right)$, considered in [14], and previously by Edge, the singular locus of $\mathrm{WM}_{f}$ consists of 40 points, consisting of 28 points giving the bitangents plus 12 points, each one corresponding to a smooth conic. This example has 12 distinct pentalateral theta.

The singular Lüroth quartics form two irreducible components $L_{1}$ and $L_{2}$ ([13]). The White-Miller quartic of a singular Lüroth quartic in $L_{2}$ (with the pentalateral having three concurrent lines) is singular along a conic in $\mathbb{P}^{5}$ corresponding to reducible conics and at seven points, each one corresponding to a smooth conic.

According to [2], pag. 368, desmic quartics have equation $f\left(x_{0}, x_{1}, x_{2}\right)=$ $\left(\left(a^{2}-2 m b c\right) x_{0}+\left(b^{2}-2 m c a\right) x_{1}+\left(c^{2}-2 m a b\right) x_{2}\right) x_{0} x_{1} x_{2}-m^{2}\left(a^{2} x_{0}^{4}+b^{2} x_{1}^{4}+\right.$ $\left.c^{2} x_{2}^{4}-2 b c x_{1}^{2} x_{2}^{2}-2 c a x_{0}^{2} x_{2}^{2}-2 a b x_{0}^{2} x_{1}^{2}\right)$ with parameters $a, b, c, m$. An explicit computation shows that $\mathrm{rk} L_{f}=14$, hence $\mathrm{rk}$ ad $L_{f}=1$ and $\mathrm{WM}_{f}$ is a double (hyper)quadric.

Klein quartic $f\left(x_{0}, x_{1}, x_{2}\right)=x_{0}^{3} x_{1}+x_{1}^{3} x_{2}+x_{2}^{3} x_{0}$ is not Lüroth, the singular locus of $\mathrm{WM}_{f}$ is just given by 28 points, as in the general case.

For the same reason, the following curve, with a cusp in $(0,0,1)$, is not Lüroth $f\left(x_{0}, x_{1}, x_{2}\right)=x_{0}^{4}+2 x_{1}^{4}-34 x_{0}^{2} x_{1}^{2}+\left(x_{0}^{2} x_{2}^{2}+x_{1}^{2} x_{2}^{2}+2 x_{0} x_{1} x_{2}^{2}\right)+41 x_{0}^{3} x_{1}+$ $51 x_{1}^{3} x_{2}+21 x_{0} x_{1}^{3}-11 x_{1} x_{2}^{3}$.

If $f$ is a Caporali quartic (sum of four 4-th powers) we have that rk $L_{f}=$ 14 and $\mathrm{WM}_{f}$ is a double (hyper)quadric.

\section{Appendix: the matrix $L_{g}$ of formula (2)}

To print the matrix $L_{g}$ is convenient to use the notation $g=x^{4} g_{0}+x^{3} y g_{1}+x^{3} z g_{2}+x^{2} y^{2} g_{3}+x^{2} y z g_{4}+x^{2} z^{2} g_{5}+x y^{3} g_{6}+x y^{2} z g_{7}+x y z^{2} g_{8}+x z^{3} g_{9}+y^{4} g_{10}+y^{3} z g_{11}+y^{2} z^{2} g_{12}+y z^{3} g_{13}+z^{4} g_{14}$

and we get 


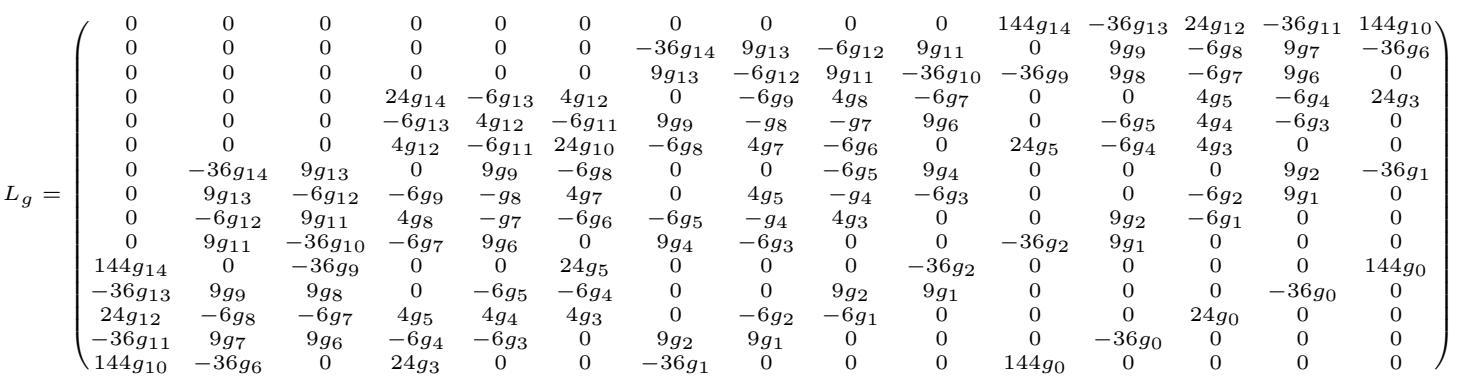

\section{References}

[1] W. Barth: Moduli of vector bundles on the projective plane, Invent. Math. 42 (1977), 63-92.

[2] H. Bateman: The Quartic Curve and its Inscribed Configurations, American J. of Math. 36 (1914), 357-386.

[3] E. Ciani, Le curve piane di quarto ordine, Giornale di Matematiche, 48 (1910), 259-304.

[4] I. Dolgachev: Classical Algebraic Geometry, a modern view , to appear in Cambridge Univ. Press.

[5] I. Dolgachev: Lectures on invariant theory, London Math. Soc. Lecture Note Series, 296, Cambridge Univ. Press, Cambridge, 2003.

[6] I. Dolgachev, V. Kanev: Polar covariants of plane cubics and quartics, Advances in Math. 98 (1993), 216-301.

[7] D. Grayson, M. Stillman, Macaulay 2: a software system for research in algebraic geometry. Available at http://www.math.uiuc .edu/Macaulay2.

[8] J.M. Landsberg, G. Ottaviani, Equations for secant varieties of Veronese and other varieties, to appear in Annali di Matematica Pura e Applicata, arXiv:1111.4567. 
[9] J. Le Potier, A. Tikhomirov, Sur le morphisme de Barth, Ann. Sci. École Norm. Sup. (4) 34 (2001), no. 4, 573-629.

[10] J. Lüroth: Einige Eigenshaften einer gewissen Gathung von Curves vierten Ordnung, Math. Annalen 1 (1868), 38-53.

[11] F. Morley: On the Lüroth Quartic Curve, American J. of Math. 41 (1919), 279-282.

[12] G. Ottaviani, E. Sernesi, On the hypersurface of Lüroth quartics, Michigan Math. J. 59 (2010), 365-394.

[13] G. Ottaviani, E. Sernesi, On singular Lüroth curves. Science China Mathematics Vol. 54 No. 8: 1757-1766, 2011, (Catanese 60th volume).

[14] D. Plaumann, B. Sturmfels, C. Vinzant, Quartic curves and their bitangents, Journal of Symbolic Computation, 46 (2011) 712-733.

[15] K. Ranestad, F.O. Schreyer, Varieties of sumes of powers, Journal für die reine und angew. Math., 525, (2000), 147-181.

[16] G. Salmon, A treatise on higher plane curves, Hodges and Smith, Dublin, 1852 (reprinted from the 3d edition by Chelsea Publ. Co., New York, 1960).

[17] G. Scorza, Un nuovo teorema sopra le quartiche piane generali, Math. Ann. 52, (1899), 457-461.

[18] B. Sturmfels, Algorithms in Invariant Theory, Springer, Wien

[19] G. Scorza, Un nuovo teorema sopra le quartiche piane generali, Math. Ann. 52, (1899), 457-461.

[20] B. Sturmfels, Algorithms in Invariant Theory, Springer, Wien, 1993.

[21] A. Tyurin, The classical geometry of vector bundles, Algebraic geometry (Ankara, 1995), Lecture Notes in Pure and Appl. Math. 193, p.347378, Dekker, New York, 1997.

[22] H.S. White, K. Miller, Note on Lüroth's type of plane quartic curves, Bull. AMS, 15, 7 (1909), 347-352.

G. OtTAVIANI - Dipartimento di Matematica "U. Dini", Università di Firenze, viale Morgagni 67/A, 50134 Firenze (Italy). e-mail: ottavian@math .unifi.it 